\title{
Evolution of Picture Archiving and Communication Systems-1989
}

\author{
Roger A. Bauman and Jaime L. Taaffe
}

\begin{abstract}
Several areas of partial picture archiving and communication systems (PACS) applications such as teleradiology, nuclear medicine, archiving modules, and systems for handling computed tomography and magnetic resonance digital data are mentioned prior to the discussion of a fully functional PACS system. Some of the present and future social implications of PACS are reviewed. Experience to date with a pilot system called MicroRSTAR at the Massachusetts General Hospital is described.

Copyright (C) 1991 by W.B. Saunders Company
\end{abstract}

KEY WORDS: computers, radiology, PACS.

$\mathbf{T}$

HIS ARTICLE addresses the development of picture archiving and communication system (PACS) today and in the future with attention to the development of such systems in the United States and elsewhere and with particular attention to work at the Massachusetts General Hospital (MGH). It was a privilege to contribute these thoughts in Sapporo, Japan, the site of a most ambitious investigation of these systems.

Efforts to realize partial or complete PACS systems are underway by a number of vendors and at a number of universities. None of the systems has yet claimed to be fully usable on the department level. The current state of the art is that pieces of PACS have been realized and are being sold and used. These pieces vary from workstations to teleradiology systems to partial PACS systems for a small portion of a department. A full PACS would handle all radiology images in a single hospital, including transmission of images between local hospitals and more distant hospitals and around the world. In the approximately 7 years that such systems have been discussed, much progress has been made. Nevertheless, realization of a truly adequate full PACS will require substantial additional work.

Teleradiology systems are a form of PACS, although the concept predates that acronym. An analog or digital image is captured and transmitted either in analog or digitized form over some media, most commonly telephone lines, to a receiving station which recreates the image on a display. A significant number of commercial vendors are active in this area. In the simplest versions, the standard telephone line is used to connect a fairly inexpensive receiver unit with the transmission station. Transmission usually requires manual handling of the images and the device at the transmitting end. Usually, the receiving end of these lower cost devices has little or no storage capability. More sophisticated units permit use of faster, dedicated telephone company lines, private lines, microwave, or satellite transmission. One of the common realities of teleradiology is the acceptance of less than ideal resolution and slower than ideal transmission speed, with the idea that the original images will be available for subsequent review. Better units are now beginning to deliver the resolution needed to be more confident of initial diagnoses. Also available now is storage in the receiving workstation, which can obviate the long transmission time of images by storing them for subsequent review by radiologists.

The impact on practice patterns by teleradiology has been felt in two principle areas: performing remote emergency interpretation in off-hour situations and in interpreting images from other locations. On-call radiologists can take their teleradiology computer/monitor receivers with them along with their beepers. By telephone line, they can access the transmission from the hospital, transport the emergency images, and render their impressions by return telephone call. This mode of operation is in use in many departments. One of the driving forces is to provide more timely coverage by radiologist. In addition to the issue of better patient care, sometimes there is also economic competition with emergency physicians who may argue that they should bill for the initial interpretation of an image.

From the Department of Radiology, Massachusetts General Hospital, Harvard Medical School, Boston, MA.

Presented at the International Symposium on HU-PACS, Sapporo, Japan, December 13-15, 1989.

Address reprint requests to Roger A. Bauman, $M D$, Department of Radiology, Massachusetts General Hospital, Harvard Medical School, Fruit St, Boston, MA 02114.

Copyright (C) 1991 by W.B. Saunders Company

0897-1889/91/0404-0102\$03.00/0 
A more extensive use of teleradiology, the transmission of images from remote hospitals or clinics, has permitted rapid interpretation of more emergent studies and even routine studies, with subsequent review of original images in many cases. Formerly, many of these places were served not at all or only infrequently by a visiting radiologist. This method of providing radiological services has been very helpful to small hospitals in the United States, especially the more sparsely settled regions.

Systems to handle nuclear medicine (NM) divisions are emerging. $\mathrm{NM}$ is ideal as a starting point, as the images are low resolution. This not only makes transmission speed and display simpler, but archiving of a significant number of images is more realistic, given present technology. An NM system could be used primarily for acquisition display and archiving or, if the additional specialized software were available, the various processing routines and calculations unique to NM could also be performed on such a system. Some of the systems interface with several nuclear gamma cameras, and thus present an opportunity to consolidate computers in NM as opposed to one computer nuclear gamma camera on which digital studies are performed.

Systems now on the market which interface with other digital acquisition devices such as computed tomography (CT), magnetic resonance imaging (MRI), or digital subtraction angiography (DSA) units are available but expensive. Often, limited archive is available, but archiving online even 1 month or several months of studies can be quite expensive. Various storage technologies, such as optical disk, VHS cassette and, possibly, optical tape archiving, are beginning to provide large volume archives, but cost remains a very real consideration. A likely application for such partial PACS systems is workstations with a minimal archive for use as a physicians' console to view CT, MRI, and or DSA studies. If these devices were reasonably priced, radiology departments could find them useful in a physician console mode to promote throughput on these expensive digital machines and to improve the lifestyle of radiologists who may now read images at unusual hours to avoid decreasing clinical throughput during the more common working hours. This in turn will provide more timely reporting of results for patient care.
Another application of a partial PACS approach which is seen likely in radiology is systems in which digital acquisition or digitization of analog images is used to create an inviolate file. This file could be used in one of two ways to distribute images in high loss areas, such as emergency wards, intensive care units, and operating rooms. First, the original radiographs could be retained in the radiology department, furnishing displays with acceptable resolution in the clinical area for clinicians to review. Second, the original images could be released before interpretation and be interpreted if promptly returned, or the computer-stored image of cases not returned by a cutoff time could be interpreted. The social and economic advantages of not losing cases to interpretation are clear.

The smaller pieces of the PACS described above fit into niches where the time demands on radiologists are not pressing, either because of low volume or because of low-resolution images. Radiologists in the United States are paid on a piecework basis. Although this might change in the future, at present radiologists do not submit a bill unless they interpret the study. The amount of work radiologists can perform in a day is limited by the speed at which they can display and interpret cases, one after the other.

The current manual system in use throughout the country is highly tuned. Although some radiologists display their own films, in many departments other personnel place these studies on multiviewers to permit the radiologist to press a foot switch and quickly display up to eight panels of images. For the complete PACS system to be widely accepted, computer workstations must present images with a speed, ease of use, and information content equal to or better than that of the current system. This is but one of several strenuous requirements that developers are working to overcome to permit the full PACS system to serve an entire department and more. But for our discussion, let us assume that such a workstation exists, and that the problems of image transmission speed, acquisition, and archiving have all been solved. What then are the implications for medicine?

A full PACS system should save space.

A full PACS system should have direct impact on patient care by reducing delays. The immediate availability of an image after acquisition by 
one of the digital acquisition devices will eliminate the time now spent manually collecting, organizing, and displaying the images for review. Comparison studies should also be available promptly in the PACS, and the computer would save the time previously spent in locating them and collating them with the new images. In welldesigned systems, radiologists would be offered only the unread cases of interest to them, automatically one after the other, for review, possible image processing, or interpretation, or to be placed on temporary hold pending resolution of some question. The possibility of selecting only one or a few images from a larger set of images for a clinician to review (or for permanent archiving), the possibility of annotating images easily, and/or the inclusion of voice messages about the case all represent possible faster means for communicating the interpretation.

A fully functional PACS should alert radiologists to other pertinent studies on the same patient. Studies from any modality should be easily displayed and reviewed. This access to any digitized or digital image from any full interpretation station raises the question of who should interpret these images. What impact will this have on subspecialization in radiology? In some departments, radiologists interpret only images of particular organs, and in other departments individual physicians specialize in CT or MRI. If a gastrointestinal (GI) radiologist does not interpret MRI studies, how will he or she benefit from seeing the MRI images pertaining to the abdomen of a patient undergoing a barium study? Will this decrease specialization in CT or MRI? The future is more clear for subspecialists who now perform roentgenography, ultrasound, CT and MRI pertaining to area of organ system specialization. The impact on others is not as clear, but the strong mitigating factor which may in the end result in little change rather than great change is that full PACS systems should also be able to display either preliminary or final interpretations of a case along with the images from that case. This would permit a GI radiologist unfamiliar with MRI to use it in correlation with the new GI study.

A full PACS system has the benefit of making available simultaneously coequal versions of an image. These images could well be displayed anywhere in the hospital, transmitted to other local area health care facilities over various transmission media or around the country and the world by satellite transmission. One social and economic dilemma that will be addressed in some way in the future is whether the role of the radiologist as primary interpreter of imaging studies is challenged. Will the emergency room physician or the orthopedic surgeon or others claim that they should now interpret and charge for interpretation for the study? The challenge to radiologists can be met in three ways: (1) greater training in skill and interpreting the images; (2) no implication of self referral; (3) rapid interpretation of the studies.

One great social change that might occur with installation of PACS is much more rapid interpretation of images because of the economic pressure brought to bear by the more widespread availability of the images. It may or may not be prudent to restrict distribution of images outside of radiology for a short period of time to permit full quality control and interpretation; long periods before release of images will probably not be well tolerated.

The fully functional PACS we have assumed for our discussion does have the capability for confident primary interpretation. This plus the archiving capability will certainly enhance teleradiology work in the future. One interesting possibility is that radiologists might compete more sharply with each other for interpretation of images. Such pressures are likely to cause radiologists to improve the speed and quality of the service they render in the future.

Other changes are also likely to benefit the patient. Integration of the fully functional PACS with radiology information systems will make more information about the patient available to the radiologist at the time of interpretation. At present, the lack of such information retards optimal efforts in helping to understand a patient's study. Interfaces with hospital information systems will extend the information base available to the radiologist even further. One of the most significant factors in speeding patient care will be the far greater availability of all imaging studies and reports to the radiologist and the clinician, making possible faster sequencing of necessary studies and avoidance of studies no longer necessary because of information already gained. The result will be not only faster diagnosis and treatment, but also dollar savings from fewer studies 
and/or shorter hospitalizations. The patient will also benefit from other computer interfaces, such as a direct interface to the pathology system. Such an interface should permit accurate and timely feedback to radiologists concerning proven disease conditions in patients they studied, which in turn will increase their diagnostic skill.

The presently available commercial systems do not yet provide a complete PACS with primary interpretation at speeds that are not a burden for radiologists. The experiment in Sapporo is magnificent, but radiologists using the system do spend some time waiting for image displays. At MGH, we have been studying the many problems of realizing a full PACS system. The project at MGH has become known as radiology storage, transfer, analysis, and reporting system (RSTAR). The following describes the realization and progress on the first-generation RSTAR system called MicroRSTAR ( $\mu$ RSTAR).

The initial $\mu$ RSTAR used a VAX 11-750 computer (Digital Equipment, Maynard, MA) with 1.3 Gbytes of disk storage as a server. The medical workstations were based on IBM AT class machines with an MGH modified display controller. Each workstation has a control monitor and one to four $512 \times 512 \times 12$ bit monitors with color graphic overlays. A two-button mouse is used, and the cursor moves easily across all screens.

During early development, CT and MRI were added to the system by magnetic tape. Positronemission tomography images were acquired directly through a digital interface. Nuclear medicine (NM) images were acquired through a inhouse-designed and -built network acquisition node that includes a floppy. disk drive.

Images are transmitted $\mu$ RSTAR workstations over standard Ethernet cable, but a special RSTAR-engineered Ethernet protocol is used (numbers 8160 and 8161). This protocol, optomized for transmission of large images, operates without interference with standard DECnet, LAT, and TCPIP protocols on the hospital Ethernet network. Image transmission times are more than 10 times faster than DECnet PCDOS. Images have been sent and received over a microwave link to the MGH Charlestown Navy Yard Research Facility through the hospital Ethernet network without impacting the network. Images have also been sent successfully by Arpanet to distant cities.

\section{Software and Function}

The software comprises over 400,000 lines of code (almost all Pascal), although standard software packages were used whenever possible.

The operational features of the system are (1) password access to the system for security and user identification; (2) expert system to manage access and configuration according to individual user privileges/category; (3) automatic presentation of unread cases sequentially to radiologists according to their work assignments (all cases or only not dictated cases); (4) automatic initial placement of study images on available display screens (and virtual screens); (5) default image display sequence determined by study type or individual radiologist preference for that particular study type; (6) initial window/centering setting accomplished automatically by a RSTAR histogram-based algorithm; (7) window/center adjustments via four preset defaults, via sliders, or via movement of mouse vertically and/or horizontally; (8) cine loops can be mixed with static images. Cine speed control and pause are available; (9) correct handling of different image sizes and shapes; (10) one, several, or all of displayed images can easily be manipulated under mouse control, including zoom and roam; flip horizontally or vertically; rotate $90^{\circ}$ either direction; change from gray scale to pseudocolor display; clear graphics/annotations; and restore image to original state. (11) multiple measurements on images with a soft display with appropriate units and optional hard copy, including distance between two points; angles; area size; point values; and regions of interest. (12) manual placement of images on workstation displays or virtual displays is done via mouse movements; (13) image processing techniques can operate on one, several, or all images. The image processing library includes the individual routines in Table $1 ;(14)$ a help function is available by general topic, via table of contents or for any mean item or icon; (15) electronic mail is implemented (useful for feedback); (16) optional research mode to retrieve particular cases, such as by patient indentifiers; by multiple key natural language simple and complex data base searches; today's cases; cases from this week; from a complete online and off- 
line archive with multiple layer cacheing uses the VAX as the image server, and from a listing of studies on the system.

$\mu$ RSTAR was intended as an interim research tool to identify and to try solutions for managing images, demographic and textual data, and workflow. As expected, actual handling of cases with $\mu$ RSTAR by a variety of users resulted in still more ideas and improvements, some of which have been implemented.

An unexpected result was pressure from radiologists to use $\mu$ RSTAR in a number of clinical situations. The operational multilayer cache for management of on-line and off-line image storage worked very well, but the initial 1.3 Gbytes of storage designated for research was not adequate to respond to this surge of clinical interest. Similarly, the clinical use placed additional strain on the entry of studies into the system. The archive remains too small, but the central server is now a VAX 6220 with 3 Gbytes of disk storage and two Gbyte optical disk drives.

Radiologists from virtually all subspecialty areas have worked with $\mu$ RSTAR, which has offered special clinical utility in generating and displaying temporal cine displays of datasets of vascular structures of MRI. A unique automated approach to quantitative bone density determinations that includes automated region of interest generation and calculation of data and control images for checking by radiologists is in daily clinical use. NM digital images of all varieties have been archived since August 1988.

The department now has six $\mu$ RSTAR workstations. Each workstation can work with any image in the central VAX archive. Display time for a $512 \times 512 \times 12$-bit image retrieved from the central archive is 2.8 seconds.

$\mu$ RSTAR was designed as a system capable of image acquisition, storage processing, measurement, and display at low cost. A deliberate design strategy was to use the off-the-shelf hardware and existing software packages and tools wherever possible. A decision was made to work with only $512 \times 512$ or smaller images, a matrix size adequate for the common digital modalities but not adequate for $\mathrm{CR}$. These decisions were based on a conviction that the first-generation $\mu$ RSTAR would be an important advance but not a fully adequate solution for primary interpretation. In addition, the speed limitations inherent in the
Table 1. Image Processing Routings

Edge enhancement by spatial mask

"Eigen Image" filtering with one desired region and up to 16 undesired regions

Performance of frequency domain image filtering with a digital Butterworth high-pass filter

Performance of frequency domain image filtering with a digital Butterworth low-pass filter

Plotting of image of power spectrum

Performance of frequency domain image filtering with a digital ideal bandpass filter

Performance frequency domain image filtering with a digital ideal bandstop filter

Performance of frequency domain image filtering with a digital ideal high-pass filter

Image processing that includes the following individual routines which use frequency domain image filtering with a digital ideal low-pass filter

Filtering out of an interactively selected region of the frequency spectrum of an image

Plotting of a power spectrum of an interactively selected region of an image

Performance of histogram normalization of an image

Creation of a new image that is a linear combination of other images

Performance of temporal matched filtering on a set of images

Combination of a collection of images into a grouping that can be viewed as an ordinary examination

Performance of mean pixel normalization on a set of images

Performance of peak neighborhood pixel normalization on a set of images

Fully automatic determination of bone density from dual-energy CT images

Creation of files of pixel data for images in a relatively portable format

Creation of histogram plots of image data

Change in raster dimensions of an image by area average interpolation

Performance of "rubber sheeting" to align corresponding anatomic features on images of two different modalities Performance of smoothing with a $3 \times 3$ spatial mask

PC and other available hardware in 1985 together with the lack of $2000 \times 2000$ cathode-ray tube (CRT) displays at reasonable cost were important factors.

The $\mu$ RSTAR project has been implemented as a complete system. The extensive body of software developed utilizes a modular approach to permit easy updating/replacement of portions of the code and to permit migration of more than $70 \%$ of the code to future generations. The inexpensive $\mu$ RSTAR hardware is viewed as "disposable."

The goal of $\mu$ RSTAR is to build a PACS that would permit identification and study of prob- 
lems and in turn allow clinicians to devise and test solutions for those problems. The radiologists using the medical workstation portion of the system continue to provide criticism and creative ideas; revised or additional features were and are being added and tried by users in iterative fashion.

$\mu$ RSTAR continues to progress, and its use and the feedback therefrom will contribute to a second-generation system to be called QRSTAR which will have $2000 \times 2500$ displays. Our progress to date has shown that the major deficiency is inadequate speed of data transmission, image processing, and image display. The small archive size is a hardware and cost problem. In addition, the user interface must be made more intuitive. QRSTAR will address the speed problems in the future. Elapsed times from a request at a QRSTAR workstation (including retrieval from the central server) to the display on the workstation are planned to be less than half a second for $2000 \times 2500$ images and less than 0.05 seconds per image for $512 \times 512$ images.

Predicting the future course and impact of PACS is difficult. Nevertheless, it is clear that many areas in the practice of medicine will be affected. Although a few of these impacts are negative and although some of them affect current specialty areas, two positive results probably will occur. The first is the most significant: better care for patients. The second is that in response to pressures created by these systems, physicians will work even harder to provide more speedy and higher quality service. 